\section{The Drosophila Ste20 family kinase dMST functions as a tumor suppressor by restricting cell proliferation and promoting apoptosis}

\author{
Jianhang Jia, ${ }^{1}$ Wensheng Zhang, ${ }_{1}^{1}$ Bing Wang, ${ }^{1}$ \\ Richard Trinko, ${ }^{2}$ and Jin Jiang ${ }^{1,3}$ \\ ${ }^{1}$ Center for Developmental Biology and Department of \\ Pharmacology and ${ }^{2} \mathrm{DCMB}$ program, University of Texas \\ Southwestern Medical Center, Dallas, Texas 75390-9133, USA
}

In a genetic screen for mutations that restrict cell growth and organ size, we identified a new tumor suppressor gene, dMST, which encodes the Drosophila homolog of the mammalian Ste20 kinase family members MST1 and MST2. Loss-of-function mutations in $d M S T$ result in overgrown tissues containing more cells of normal size. dMST mutant cells exhibit elevated levels of Cyclin E and DIAP1, increased cell growth and proliferation, and impaired apoptosis. dMST forms a complex with Sav and Wts, two tumor suppressors also implicated in regulating both cell proliferation and apoptosis, suggesting that they act in common pathways.

Received July 16, 2003; revised version accepted August 25, 2003.

In animal development, cell number and organ size are tightly controlled by mechanisms that regulate cell proliferation and cell death. Deregulation of these mechanisms has been implicated in cancers (Hanahan and Weinberg 2000). Although many genes controlling either cell proliferation or cell death have been identified, few genes have been implicated in regulating both processes. One exception is the pathway defined by the two genes salvador (sav) and warts (wts)/large tumors (lats), which encode WW domain-containing protein and Ser/Thr kinase, respectively (Justice et al. 1995; Xu et al. 1995; Tapon et al. 2002). Mosaic animals carrying sav or wts mutant clones exhibit tissue overgrowth phenotypes. Both sav and lats mutant cells contain elevated levels of Cyclin E and Drosophila inhibitor of apoptosis 1 (DIAP1), leading to increased cell proliferation and impaired apoptosis (Tapon et al. 2002). Sav binds Wts in vitro, and they genetically interact, suggesting they act in a common pathway to regulate cell proliferation and cell death. How the sav/wts pathway is regulated and the mechanisms by which it controls cell proliferation and apoptosis are not known.

[Keywords: dMST; MST1; tumor suppressor; apoptosis; Wts; Sav] ${ }^{3}$ Corresponding author.

E-MAIL jin.jiang@utsouthwestern.edu; FAX (214) 648-1960.

Article and publication are at http://www.genesdev.org/cgi/doi/10.1101/ gad.1134003

\section{Results and Discussion}

To identify novel tumor suppressor genes, we systematically screened the Drosophila genome for mutations that cause tissue overgrowth phenotypes (see Materials and Methods). From this screen, we identified three alleles of a gene we named $d M S T$ (Drosophila homolog of MST1 and MST2). We used $d M S T^{B F 33}$ for most analyses described in this study, as the molecular nature of $d M S T^{B F 33}$ suggests that it is likely to be a null allele (see below).

\section{dMST mutations result in tumor-like growth}

Compared with wild-type eyes, $d M S T$ mosaic eyes are significantly larger and often protrude out in folds (Fig. 1A,B). Tumorous outgrowths were also observed when dMST clones were induced in other places, including the thorax, wing, and haltere (Fig. 1C-G). Hence, dMST is generally required for restricting tissue growth and organ size.

To determine how $d M S T$ controls organ size, we generated labeled $d M S T$ clones in wing discs and compared cell size and clone size between mutant clones and twin spots. dMST mutant cells do not exhibit discernible changes in cell size (Fig. 1J-K'). Moreover, $d M S T$ mutant cells differentiated into wing margin bristles of normal size (Fig. 1H). However, dMST clones occupy significantly larger areas and contain more cells than do wildtype twin spots (Fig. 1I, $\left.\mathrm{I}^{\prime}\right)$. As mutant clones and twin spots were derived from mitotic sister cells born at the same developmental stages, the increase in cell numbers and tissue mass of $d M S T$ mutant clones over twin spots suggests that $d M S T$ mutant cells grow and proliferate faster than do wild-type cells. Hence, the increase in size of $d M S T$ mosaic organs is caused by an increase in cell number but not cell size.

\section{dMST regulates both cell proliferation and apoptosis}

To further explore cell proliferation defects caused by dMST mutations, we focused on eye development. In wild-type eye discs, a single stripe of cells, referred to as the second mitotic wave (SMW), enters S phase synchronously posterior to the morphogenetic furrow (MF), and little bromodeoxyuridine (BrdU) labeling is present posterior to the SMW (Fig. 2A; Wolff and Ready 1993). In contrast, $d M S T$ mosaic discs exhibit extensive BrdU incorporation posterior to the SMW (Fig. 2B). To determine if extra mitosis also occurs in $d M S T$ mutant eye discs, we used the anti-phosphohistone $\mathrm{H} 3(\mathrm{pH} 3)$ antibody to label cells in $\mathrm{M}$ phase. In wild-type eye discs, few cells posterior to the SMW exhibit pH3 staining (Fig. 2C). In contrast, $d M S T$ mutant discs contain increased number of cells in M phase posterior to the SMW (Fig. 2D), suggesting that $d M S T$ mutations increase cell proliferation.

Cyclin E is an important regulator of S-phase initiation and progression in imaginal disc development (Richardson et al. 1995). In wild-type discs, Cyclin E is up-regulated at the SMW (Fig. 2E). We found that $d M S T$ mutant clones accumulate high levels of Cyclin $\mathrm{E}$ in a cell-autonomous fashion (Fig. 2F-F"), which is consistent with the increased cell proliferation in $d M S T$ mutant discs.

In wild-type eyes, excessive cells between differentiated ommatidias are eliminated by a wave of apoptosis at 

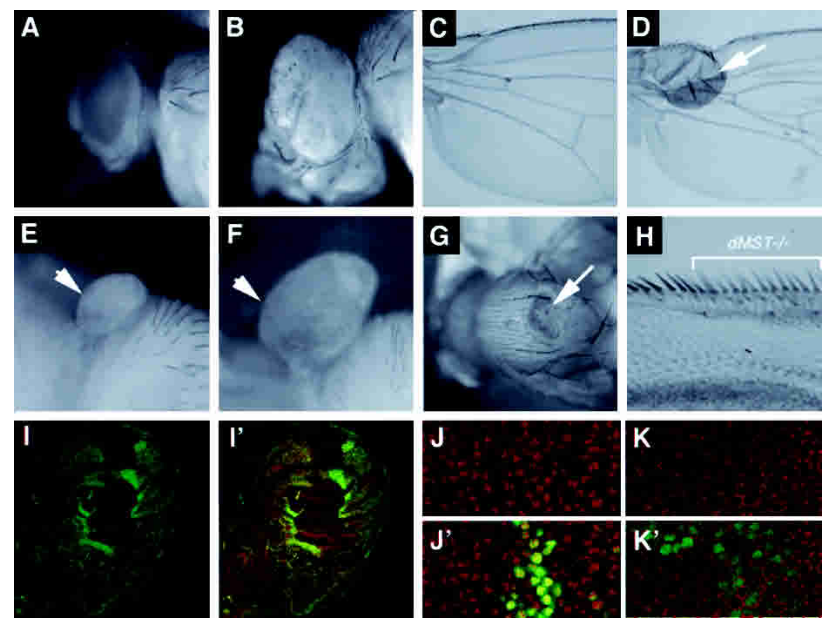

Figure 1. $d M S T$ mutations result in tumorous overgrowths. $(A, B)$ Adult eyes of wild type $(A)$ or containing $d M S T$ mutant clones $(B)$. (B) dMST mosaic eye is enlarged and protrudes out in folds. $(C, D) \mathrm{A}$ wild-type wing $(C)$ or a wing containing a $d M S T$ clone located near the wing hinge (arrow in $D) .(E, F)$ A control $(E)$ and a $d M S T$ mosaic haltere $(F)$. $(G)$ A $d M S T$ mutant clone on the thorax exhibits tumorlike growth (arrow). (H) High-magnification view of wing margin containing dMST mutant clones labeled by $y-$ (indicated by the bracket). dMST mutant cells differentiate into margin bristles of normal size. $\left(I, I^{\prime}\right)$ A late third instar wing disc containing dMST clones stained with a nuclear dye, 7-AAD (red in $I^{\prime}$ ) and anti-Myc antibody (green in $I, I^{\prime}$ ). dMST mutant clones lack hs-Myc-GFP expression, whereas the twin spots exhibit enhanced hs-Myc-GFP expression. (J-K') High-magnification view of $d M S T$ mutant clones and twin spots labeled with 7-AAD (red in $\left.J, J^{\prime}\right)$, which labels the nuclei, or anti-Armadillo (Arm) antibody (red in $\left.K, K^{\prime}\right)$, which stains cell membrane. $d M S T$ mutant cells are recognized by the lack of Myc-GFP expression (green). $d M S T$ mutant cells are of the normal size compared with wild-type cells.

early pupal stage so that a single layer of cells exists between two adjacent ommatidias (Fig. 2G; Wolff and Ready 1993). In contrast, the dMST mutant disc contains multiple layer interommatidial cells (Fig. 2H). The persistence of excessive interommatidial cells in $d M S T$ mutant discs implies that apoptosis could be compromised. To test this, we examined cell death in wild-type and dMST mosaic pupal retina $38 \mathrm{~h}$ after pupa formation (APF) and found that apoptosis was diminished in $d M S T$ mutant cells (Fig. 2I-I"). Hence, $d M S T$ is required for apoptosis during development.

In Drosophila, apoptosis is triggered by death inducers, including head involution defective (hid; Abrams 1999). Expressing GMR-hid induces precocious cell death in larval eye discs (Fig. 2J), which is blocked in $d M S T$ mutant cells (Fig. $2 \mathrm{~K}, \mathrm{~K}^{\prime}$ ). As a consequence, adult eyes derived from $G M R$-hid-expressing discs that also contain dMST mutant clones are larger than those derived from wild-type discs expressing GMR-hid (Fig. 2L,M). In Drosophila, death promoters induce apoptosis in part by down-regulating the levels of the death inhibitor DIAP1 (Ryoo et al. 2002; Yoo et al. 2002). We found that dMST mutant cells exhibit higher levels of DIAP1 than do wild-type cells (Fig. 2N-O'), suggesting that $d M S T$ promotes cell death at least in part by down-regulating the levels of DIAP1. We also found that the expression of a diap1-lacZ reporter gene is elevated in $d M S T$ mutant cells (Fig. 2Q, $\mathrm{Q}^{\prime}$ ), indicating that $d M S T$ inhibits the transcription of diap1.

\section{Molecular cloning of dMST}

The dMST mutations were mapped by high-resolution meiotic recombination (Fig. 3A; see Materials and Methods). We sequenced several candidate genes for molecular lesions present in mutagenized chromosomes containing $d M S T$ alleles. Both $d M S T^{I M 1}$ and $d M S T^{B F 33}$ in-

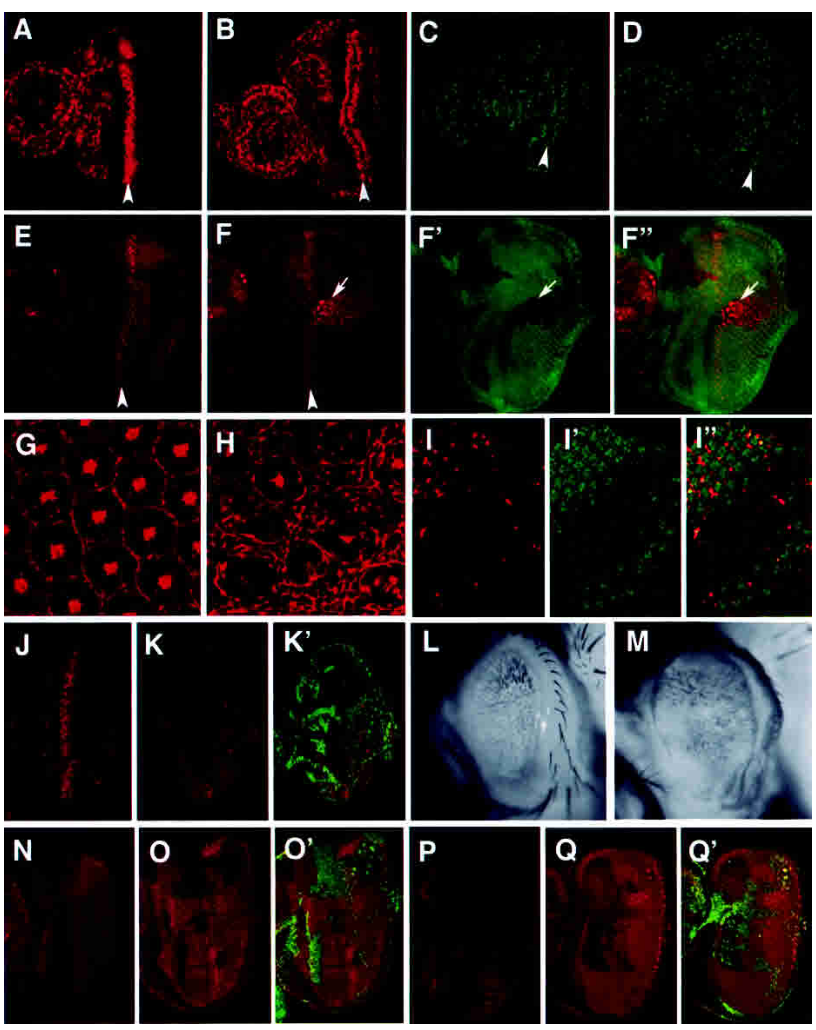

Figure 2. $d M S T$ controls both cell proliferation and apoptosis. $(A-D)$ BrdU incorporation $(A, B)$ or phospho-H3 staining $(C, D)$ of wild-type eye discs $(A, C)$ or $d M S T$ mosaic eye discs $(B, D)$. Arrowheads indicate the second mitotic wave (SMW). All eye discs are oriented with anterior to the left. $(B, D)$ dMST mosaic discs exhibit extensive BrdU incorporation and increased phospho-H3-positive cells posterior to the SMW. (E) Cyclin E expression in wild type. $\left(F-F^{\prime \prime}\right)$ Cyclin E expression in dMST mosaic discs. dMST mutant cells are labeled by the lack of Myc-GFP expression (green). dMST mutant cells posterior to the SMW accumulate high levels of Cyclin E cell autonomously (arrows). $(G, H)$ Phalloidin staining of wild type $(G)$ or $d M S T$ mosaic $(H)$ pupal eye disc (46 h APF). $(G)$ The wild-type pupal eye disc contains a single layer of interommatidial cells. $(H)$ In contrast, the $d M S T$ mutant disc contains multiple layers of interommatidial cells. (I- $\left.I^{\prime \prime}\right)$ A $d M S T$ mosaic pupal eye disc (38 h APF) doubly stained to show TUNEL (red) and Myc-GFP (green) expression. dMST mutant cells are labeled by the lack of green staining. The TUNEL staining is confined to wild-type cells. $(J)$ A late third eye disc expressing GMR-hid and stained with an antibody against an activated form of the Drosophila caspase Drice (Drice-Act), which labels dying cells (Yoo et al. 2002). Expression of GMR-hid induces precocious cell death. $\left(K, K^{\prime}\right)$ A $d M S T$ mosaic eye disc expressing GMR-hid stained with antibodies against Drice-Act (red) and Myc (green). dMST mutant cells are recognized by the lack of green staining. Ectopic cell death induced by GMR-hid is blocked in $d M S T$ mutant cells. $(L, M)$ Adult eyes derived from $G M R$-hid-expressing discs $(L)$ or dMST mosaic discs expressing GMR-hid $(M)$. (N-Q) Diap1 protein accumulation (red in $N-\mathrm{O}^{\prime}$ ) or diap1-1acZ expression (red in $\left.P-Q^{\prime}\right)$ in wild-type $(N, P)$ or $d M S T$ mosaic $\left(O, O^{\prime}, Q_{,} Q^{\prime}\right)$ eye discs. dMST mutant cells, which lack Myc-GFP expression (green), exhibit high levels of DIAP1 protein $\left(O, O^{\prime}\right)$ and diap1-lacZ expres$\operatorname{sion}\left(Q, Q^{\prime}\right)$. 
Jia et al.

A

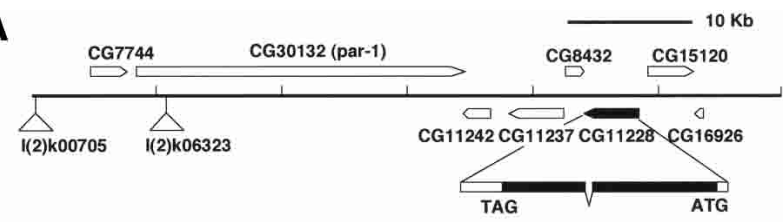

B

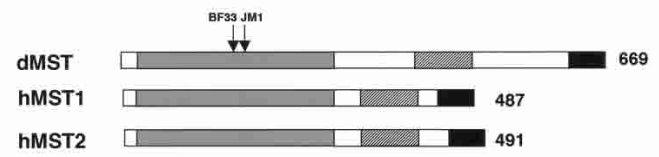

C

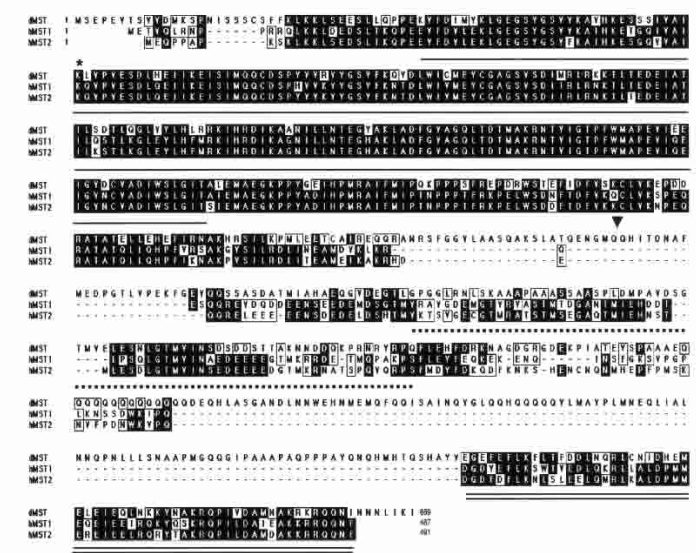

Figure 3. $d M S T$ encodes the homolog of the mammalian Ste20-like kinases MST1 and MST2. (A) The genomic region of the dMST locus. The positions of two of the P-element insertions used for mapping-l(2)k06323 and l(2)k00705-are indicated. (B) Functional domains of dMST, and human MST1 (hMST1) and MST2 (hMST2). The kinase domains are shaded in gray. The black and hatched boxes indicate the dimerization and kinase inhibitory domains, respectively. The arrows indicate the positions of $d M S T^{B F 33}$ and $d M S T^{I M 1}$ mutations. (C) Sequence alignment among dMST, hMST1, and hMST2. The kinase domain, kinase inhibitory domain, and dimerization domain are underlined by a single solid line, a broken line, and a double solid line, respectively. The asterisk indicates K71, which is changed to $\mathrm{R}$ in $\mathrm{dMSTnK}>\mathrm{R}$ mutant. The arrowhead marks the boundary between dMSTn and dMSTc.

troduced point mutations in the open reading frame (ORF) of an annotated gene CG11228, which encodes the Drosophila homolog of mammalian Ste20 kinase family members MST1 and MST2 (Creasy and Chernoff 1995a,b). A full-length cDNA corresponding to CG11228 was placed under the control of a tubulin $\alpha 1$ promoter to generate tub-dMSTf. Both $d M S T^{I M 1}$ and $d M S T^{B F 33}$ homozygotes expressing one copy of tub-dMSTf are viable, morphologically normal, and fertile, demonstrating that CG11228 is dMST. dMST $T^{I M 1}$ introduced a single amino acid substitution at a conserved residue (G181E), whereas $d M S T^{B F 33}$ introduced a stop codon at amino acid 174 (Fig. $3 \mathrm{~B}$ ), suggesting that $d M S T^{B F 33}$ is likely to be a null allele.

Like its mammalian counterparts, dMST contains an $\mathrm{N}$-terminally situated kinase domain and C-terminally located regulatory domains (Fig. 3B,C). The dMST kinase domain exhibits $84 \%$ and $83 \%$ identity to that of hMST1 and dMST2, respectively. The C-terminally situated dimerization and kinase inhibitory domains present in MST1 and MST2 (Creasy et al. 1996) are also highly conserved in dMST (Fig. 3C).

\section{dMST interacts with Sav and Wts}

The dMST mutant phenotypes closely resemble those caused by sav or wts mutations (see Fig. 5I, I', L-L",O-O", below; Tapon et al. 2002; data not shown). It has been shown that Sav and Wts physically and genetically interact, suggesting that they may act in common pathways (Tapon et al. 2002). To determine if dMST could act in the same pathways, we examined if it physically interacts with Sav and Wts by using coimmunoprecipitation assay. We first determined if dMST binds Sav. S2 cells were transfected with DNA constructs expressing HA-tagged Sav and Flag-tagged full-length dMST (dMSTf), its $\mathrm{N}$-terminal fragment containing the kinase domain (dMSTn), or its C-terminal fragment containing the regulatory domains (dMSTc; Fig. 4A). As shown in Figure 4B, both dMSTf and dMSTc, but not dMSTn, were coimmunoprecipitated with Sav, suggesting that dMST binds Sav through its C-terminal regulatory region. The dimerization domain at the $\mathrm{C}$ terminus of dMST appears to be essential for interaction as deletion of this domain from dMSTc abolished its ability to bind Sav (data not shown).

To define the domain in Sav that binds dMST, we gen-

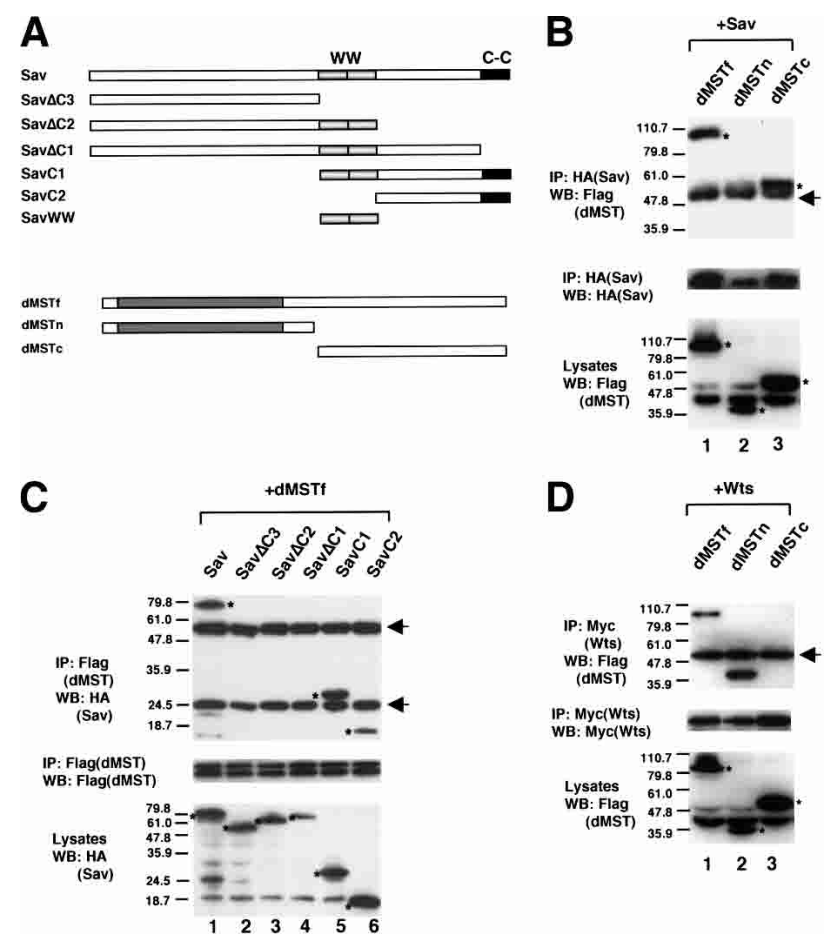

Figure 4. dMST interacts with Sav and Wts. $(A)$ Schematic drawing of Sav and dMST constructs used for transfection. The WW and coiled-coil (C-C) domains of Sav are indicated by shaded and black boxes, respectively. The Sav constructs contain two copies of HA tag, whereas the dMST constructs have a Flag tag at the N terminus. $(B-D)$ S2 cells were transfected in indicated DNA constructs. (Top and middle panels) Cell extracts were immunoprecipitated (IP) with indicated antibodies, followed by Western blot (WB) with corresponding antibodies, as indicated. (Bottom panels) Cell lysates were also directly subjected to Western blot with indicated antibodies to determine the expression levels of individual constructs. The Wts construct contains six copies of Myc tag at the $\mathrm{N}$ terminus. The asterisks indicate the positions of proteins expressed from the transfected constructs (bottom panels) or coimmunoprecipitated proteins (top panels). The arrows indicate immunoglobin G. Of note, dMSTf and dMSTc appear to be much larger than their predicted sizes. 
erated a series of truncated forms of Sav (Fig. 4A). Both C-terminal fragments, SavC1 and SavC2, bind dMST. In contrast, all the C-terminally truncated fragments, including $\operatorname{Sav} \Delta \mathrm{C} 1, \operatorname{Sav} \Delta \mathrm{C} 2$, and Sav $\Delta \mathrm{C} 3$, fail to bind dMST (Fig. 4C), suggesting that dMST binds the C-terminal region of Sav and the coiled-coil domain of Sav is essential.

We next examined if dMST binds Wts. S2 cells were transfected with DNA constructs expressing Myc-tagged Wts and Flag-tagged dMSTf, dMSTn, or dMSTc. As shown in Figure 4D, Wts binds dMSTf and dMSTn, but not dMSTc, suggesting that Wts interacts with the $\mathrm{N}$ terminal region of dMST. The interaction between Wts and dMST is not affected by Sav, as coexpression of Sav does not increase the amount of dMSTf coimmunoprecipitated with Wts (data not shown). However, it remains possible that Sav might regulate dMST/Wts interaction in vivo at physiological concentration. Taken together, these results suggest that dMST, Wts, and Sav form a complex in which Wts and Sav bind the kinase and regulatory domains of dMST, respectively.

When overexpressed in larval eye discs by using the GMR-gal4 driver, dMSTn, but not its kinase dead form $(\mathrm{dMSTnK}>\mathrm{R})$, induced ectopic apoptosis (Fig. 5E,F), leading to the formation of small rough eyes (Fig. 5B). Although expressing GMR-Wts alone does not cause any significant defect (Fig. 5C), coexpressing GMR-Wts with dMSTn enhanced the defects caused by expressing dMSTn alone. For example, although flies expressing GMR-Gal4/dMSTn are viable, coexpressing GMR-Wts with GMR-gal4/dMSTn causes flies to die as pharate adults that have smaller eyes than those of flies expressing GMR-gal4/dMSTn alone (Fig. 5D). Similar phenotypic enhancement was obtained when two copies of $U A S-d M S T n$ were expressed under GMR-gal4 (data not shown). In contrast, the defects caused by dMSTn are not enhanced by GMR-Sav (data not shown). These genetic interactions are consistent with our findings that dMSTn binds Wts but not Sav.

To determine the genetic epistasis, we overexpressed dMSTn with GMR-gal4 in eye discs that contain wts or sav mutant clones. dMSTn induces ectopic Drice activation in sav mutant cells, as in the case of wild-type cells (Fig. 5G, $\mathrm{G}^{\prime}$ ). In contrast, the ectopic Drice activation induced by dMSTn is diminished in wts mutant cells (Fig. 5H, $\mathrm{H}^{\prime}$ ). Consistently, dMSTn blocks the upregulation of DIAP1 in $s a v$, but not in wts, mutant cells (Fig. 5J-K'). In addition, we found that dMSTn blocks the up-regulation of Cyclin $\mathrm{E}$ in sav but not in wts mutant cells (Fig. 5M'; $\mathrm{M}^{\prime \prime}, \mathrm{P}-\mathrm{P}^{\prime \prime}$ ). These results suggest that $\mathrm{dMST}$ acts downstream of sav but upstream of or in parallel with wts to restrict cell proliferation and promote apoptosis.

\section{Conclusion and perspective}

The MST subfamily of Ser/Thr kinases is classified as putative mitogen-activated protein kinase kinase kinase kinase (MAP4K; Dan et al. 2001). Although numerous studies have been carried out to address their biochemical properties and regulations in cultured cells (Creasy and Chernoff 1995b; Creasy et al. 1996; Hirai et al. 1997; Graves et al. 1998; Lee et al. 1998; Glantschnig et al. 2002; Lee and Yonehara 2002; Cheung et al. 2003), their physiological roles remain elusive. In this study, we demonstrated that dMST plays a pivotal role in controlling cell number and organ size in Drosophila develop-
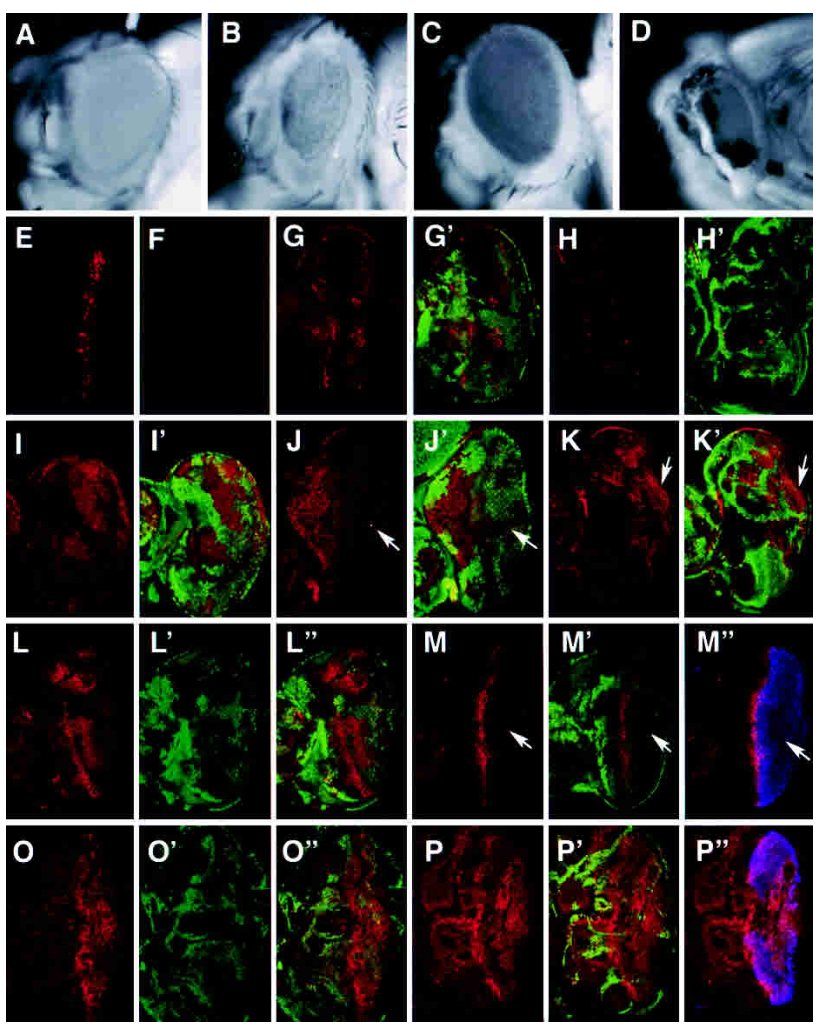

Figure 5. Genetic interaction among $d M S T$, sav, and wts. $(A-D)$ Adult eyes of wild type $(A)$, GMR-gal4/UAS-dMSTn $(B)$ GMR-Wts (C), and GMR-gal4/UAS-dMSTn;GMR-Wts (D). (E,F) Drice-Act staining in eye discs expressing GMR-gal4/UAS$d M S T n(E)$ or $G M R$-gal4/UAS-dMSTnKR $(F) .\left(G-H^{\prime}\right)$ Drice-Act staining (red) in eye discs expressing GMR-gal4/UAS-dMSTn and containing $\operatorname{sav}\left(G, G^{\prime}\right)$ or wts $\left(H, H^{\prime}\right)$ mutant clones. sav or wts mutant cells are labeled by the lack of Myc-GFP staining (green). $\left(I-I^{\prime}\right)$ DIAP1 expression (red) in sav mosaic eye disc $\left(I, I^{\prime}\right)$ or sav mosaic eye disc expressing GMR-gal4/UAS-dMSTn $\left(J, J^{\prime}\right)$. sav mutant cells are labeled by the lack of Myc-GFP staining (green). dMSTn blocks the up-regulation of DIAP in sav mutant cells (arrow). $\left(K, K^{\prime}\right)$ DIAP1 expression in wts mosaic disc expressing GMR-gal4/UAS-dMSTn. wts mutant cells (labeled by the lack of green staining) exhibit high levels of DIAP1 even though they express GMR-gal4/UAS-dMSTn (arrow). $\left(L-P^{\prime \prime}\right)$ Cyclin E staining (red) in sav $\left(L-L^{\prime \prime}\right)$, wts $\left(O-O^{\prime \prime}\right)$ mosaic discs, or sav mosaic disc expressing GMR-gal4/UAS-dMSTn $\left(M-M^{\prime \prime}\right)$, or mosaic disc expressing GMR-gal4/UAS-dMSTn $\left(P-P^{\prime \prime}\right)$. $s a v$ and wts mutant cells are labeled by the lack of Myc-GFP staining (green). dMSTn expression is indicated by Flag staining (blue). sav mutant cells accumulate high levels of Cyclin E cell autonomously $\left(L-L^{\prime \prime}\right)$, which is suppressed by dMSTn (arrows in $M-M^{\prime \prime}$ ). $\left(P-P^{\prime \prime}\right)$ In contrast, wts mutant cells accumulate high levels of Cyclin E even though they express dMSTn.

ment. We showed that dMST fulfills such a role both by restricting cell growth and proliferation and by promoting cell death. The cell-autonomous elevation of Cyclin $\mathrm{E}$ and DIAP1 levels in $d M S T$ mutant clones suggest that dMST regulates cell proliferation and cell death cell autonomously. We provide biochemical evidence that dMST, Sav, and Wts form a complex. Our genetic epitasis study suggests sav acts upstream of dMST whereas wts downstream of or in parallel with dMST. Sav could regulate the formation or activity of $\mathrm{dMST} / \mathrm{Wts}$ kinase complex. Alternatively, Sav could act to bridge the $\mathrm{dMST} / \mathrm{Wts}$ kinase complex to its substrates, a function that can be bypassed by excess amounts of dMSTn.

It should be noted that $s a v, d M S T$, and wts may not 
simply act in a linear pathway, as wts mutant phenotypes appear to be more severe than those caused by sav or dMST mutations (data not shown). In addition, sav wts double-mutant phenotypes are stronger than sav or wts single-mutant phenotypes (Tapon et al. 2002). Hence, although our results suggest that dMST, Sav, and Wts act in common pathways, they may exist in multiple complexes and have independent functions.

It has been shown that human Sav is mutated in several cancer cell lines, and mice lacking a homolog of wts/lats develop hyperplasia and tumors in several tissues (St. John et al. 1999; Tapon et al. 2002), raising the possibility that mammalian MST1 and MST2 may also function as tumor suppressors. In support of this notion, MST1 and MST2 have been implicated in promoting cell death in cultured mammalian cells (Graves et al. 1998; Lee et al. 2001; Cheung et al. 2003). It remains to be determined whether MST1 and MST2 also regulate cell proliferation in mammals. Given the functional conservation between many insect and mammalian pathways, we speculate that the mammalian MST/Sav/Lats pathway may also participate in restricting cell number and organ size during normal development, and its malfunction may lead to cancers.

\section{Materials and methods}

Mutations and transgenes

The scheme for genetic screen using the eyFLP system has been described (Newsome et al. 2000; Amanai and Jiang 2001). For 2R and 3R screen, isogenic FRT-containing males (FRT42D and FRT82B) were mutagenized with $25 \mathrm{mM}$ ethylmethanesulfonate (EMS). After $24 \mathrm{~h}$ recovery, the males were mated with female virgins with corresponding eyFLP/FRT (y w eyFLP2; FRT42D $\mathrm{w}+1[2] \mathrm{cl}-R 1 / C y o[\mathrm{y}+]$ for $2 \mathrm{R}$ or $\mathrm{y} w$ eyFLP2 glass-lacZ; FRT82B $w+$ cl3R3/TM6B, $y+$ for $3 \mathrm{R})$. The $\mathrm{F}_{1}$ progenies exhibiting enlarged eyes were back-crossed to the eyFLP/FLP stocks for verifying the phenotypes and germline transmission. The $F_{2}$ progenies that retained the overgrowth phenotypes were crossed to the balancer stock, y w; Sp/Cyo $/ \mathrm{y}+] ; T M 2 / T M 6 B$ to establish lines. Three alleles of $d M S T\left(d M S T^{I M 1}, d M S T^{I M 4}\right.$, and $\left.d M S T^{B F 33}\right)$, two alleles of $\operatorname{sav}\left(\mathrm{sav}^{S H 8}\right.$ and $\left.s a v^{S H 13}\right)$, and three alleles of $w t s\left(w t s^{R 23}, w t s^{S H 15}\right.$, and $\left.w t s^{S H 16}\right)$ were isolated. By using a combination of meiotic recombination and complementation test with the $2 \mathrm{R}$ deficiency kit, $d M S T$ mutations were mapped to a gap (56C11-56F5) between two nonoverlapping deficiencies $D f(2 R) P 34$ (55E2-56C11) and $D f(2 R) 017$ (56F5-56F15). Fine mapping was achieved by high-resolution meiotic recombination by using several $\mathrm{P}$ element insertion lines in this region. Specifically, $d M S T$ is $0.062 \%$ (8/12898) distal to $1(2) \mathrm{k} 06323$ (BS\#10615), 0.059\% (10/17011) distal to l(2)k00705 (BS\#10485), and $0.072 \%(12 / 16488)$ distal to $1(2) \mathrm{k} 06121$ (BS\#10607). dMST is placed distal to 1(2) $\mathrm{k} 06323$ and $1(2) \mathrm{k} 06121$ because the majority of the recombinant chromosomes between $d M S T$ and the $\mathrm{P}$ elements retained FRT42D. Several candidate genes situated $30-100 \mathrm{~kb}$ distal to $1(2) \mathrm{k} 06323$ insertion site were chosen for sequencing. $d M S T$ alleles were balanced over a GFP balancer. Both $d M S T^{I M 1}$ and $d M S T^{B F 33}$ homozygotes die at early larval stages. Genomic DNA was prepared from homozygous embryos and amplified by PCR, followed by direct sequencing. Both $d M S T^{I M 1}$ and $d M S T^{B F 33}$, but not $d M S T^{I M 4}$, contained a point mutation in the coding region of CG11228 (Fig. 3).

$d M S T$ full-length cDNA was obtained from Research Genetics (GH10354) and was verified by sequencing. The coding sequence for the full-length, the N-terminal fragment (1-342 amino acids), or the C-terminal fragment (343-669 amino acids) of dMST was PCR-amplified and subcloned into the pUAST-Flag vector to generate UAS-dMSTf, UASdMSTn, and UAS-dMSTc. All constructs contain a Flag tag fused in frame to the $\mathrm{N}$ terminus. UAS- $d M S T n K R$ contains a single amino acid substitution at position 71 (K71R) generated by PCR-based site-direct mutagenesis. tub-dMST contains a 2.6-kb tubulina1 promoter fused to dMST full-length cDNA in Casper vector (Basler and Struhl 1994). sav full-length cDNA was obtained from Research Genetics (SD10307). The coding sequence corresponding to various regions of Sav was PCR-am- plified and fused in frame with two copies of HA tags, followed by subcloning into the $p U A S T$ vector to generate UAS-HA-Sav (amino acids 1-608), UAS-HA-SavC1 (amino acids 439-608), UAS-HA-SavC2 (amino acids 507-608), UAS-HASavWW (amino acids 440-506), UAST-HASav $\triangle C 1$ (amino acids 1-591), UAST-HA-Sav $\Delta C 2$ (amino acids 1-507), and UAST-HA-SavDC3 (amino acids 1-439). UAS-Myc-Wts contains six copies of Myc tag at the $\mathrm{N}$ terminus of Wts. Other transgenes and stocks used in this study are diap-lacZ ( $t h^{i 5 c 8}$; Ryoo et al. 2002), hs-Myc-GFP (Jiang and Struhl 1998), hs-CD2 (Jiang and Struhl 1995), GMR-Gal4 (Freeman 1996), GMR-hid (Stowers and Schwarz 1999), GMR-sav, and GMRwts (Tapon et al. 2002). sav ${ }^{4}$ is a strong allele (Tapon et al. 2002). wts $^{M 72}$ is a strong allele isolated in a previous screen using the hsFLP/FRT system (Jiang and Struhl 1995).

Generation of clones of mutant cells

$d M S T$ mutant clones were generated using FLP/FRT-mediated mitotic recombination as described previously (Jiang and Struhl 1995). The genotypes for generating $d M S T$ mosaic eyes with ey-FLP: eyFLP2/+ or $Y$; FRT42D w+ 1(2) cl-R1/FRT42D dMST, or eyFLP2/+ or Y; FRT42D hsMyc-GFP/FRT42D dMST. The genotype for generating $d M S T$ clones with hs-FLP: hs-flp122/+ or Y; FRT42D hs-Myc-GFP/FRT42D dMST or hs-flp122/+ or Y; FRT42D hs-CD2 y+/FRT42D dMST. For generating $d M S T$ mutant eyes expressing hid, the following genotype is used: eyeflp/GMR-hid; FRT42D hs-Myc-GFP/FRT42D dMST. To generate sav or wts mosaic eyes with dMSTn expression, the following genotypes were used: eye-flp/+ or Y; GMR-hid/UAS-dMSTn; FRT82B hs-Myc-GFP/ FRT82B $\operatorname{sav}^{4}\left(\right.$ wts $\left.^{M 72}\right)$.

Immunohistochemistry

Standard protocols for immunofluorescence staining, phalloidin staining, BrdU labeling, and TUNEL assay were used (Jiang and Struhl 1998; Wolff 2000). Primary antibodies used in this study are rat anti-CycE (Richardson et al. 1995), mouse anti-phosphohistone H3 (Upstate Biotechnology), mouse and rabbit anti-Diapl (Ryoo et al. 2002; Yoo et al. 2002), rabbit anti-DriceAct (Yoo et al. 2002), rabbit anti- $\beta$ Gal (Cappel), mouse antiMyc (Santa Cruz Biotechnology), rabbit anti-GFP (Clone Tech), and mouse anti-Arm (Jiang and Struhl 1998). Nuclear dye, 7-AAD, is from Molecular Probes.

Cell culture and immunoprecipitation

S2 cells were cultured in the Schneider's Drosophila Medium (Invitrogen) with $10 \%$ fetal bovine serum, $100 \mathrm{U} / \mathrm{mL}$ of penicillin, and $100 \mu \mathrm{g} /$ $\mathrm{mL}$ of streptomycin. Transfection was carried out by using the Calcium Phosphate Transfection Kit (Specialty Media) according to manufacturer's instructions. To express dMST and Sav in S2 cells, corresponding UAS constructs were cotransfected with an ub-Gal4-expressing construct. Immunoprecipitation and Western blot analysis were performed by using standard protocols as previously described (Robbins et al. 1997). Antibodies used are mouse anti-Myc (Santa Cruz), mouse anti-HA, F7 (Santa Cruz), and mouse anti-Flag, M2 (Sigma).

\section{Acknowledgments}

We thank Jason Mercer, Maryam Shansab, and Brenda Fortney for conducting genetic screen, and Liping Luo for technical assistance. We thank Drs. Bruce Hay, Iswar Hariharan, H.D. Ryoo, Wei Du, and H. Richardson and the Bloomington stock center for reagents and fly stocks. J.J is supported by a grant from NIH, Searle Scholar Program from Chicago Trustee, Leukemia and Lymphoma Society Scholar Program, and Endowed Scholar Program in Biomedical Science from University of Texas Southwestern Medical Center.

The publication costs of this article were defrayed in part by payment of page charges. This article must therefore be hereby marked "advertisement" in accordance with 18 USC section 1734 solely to indicate this fact.

\section{References}

Abrams, J.M. 1999. An emerging blueprint for apoptosis in Drosophila. Trends Cell. Biol. 9: 435-440.

Amanai, K. and Jiang, J. 2001. Distinct roles of central missing and dispatched in sending the Hedgehog signal. Development 128: 51195127. 
Basler, K. and Struhl, G. 1994. Compartment boundaries and the control of Drosophila limb pattern by hedgehog protein. Nature 368: 208214.

Cheung, W.L., Ajiro, K., Samejima, K., Kloc, M., Cheung, P., Mizzen, C.A., Beeser, A., Etkin, L.D., Chernoff, J., Earnshaw, W.C., et al. 2003. Apoptotic phosphorylation of histone H2B is mediated by mammalian sterile 20 kinase. Cell 113: 507-517.

Creasy, C.L. and Chernoff, J. 1995a. Cloning and characterization of a human protein kinase with homology to Ste20. J. Biol. Chem. 270: 21695-21700.

- 1995b. Cloning and characterization of a member of the MST subfamily of Ste20-like kinases. Gene 167: 303-306.

Creasy, C.L., Ambrose, D.M., and Chernoff, J. 1996. The Ste20-like protein kinase, Mst1, dimerizes and contains an inhibitory domain. $J$. Biol. Chem. 271: 21049-21053.

Dan, I., Watanabe, N.M., and Kusumi, A. 2001. The Ste20 group kinases as regulators of MAP kinase cascades. Trends Cell. Biol. 11: 220-230.

Freeman, M. 1996. Reiterative use of the EGF receptor triggers differentiation of all cell types in the Drosophila eye. Cell 87: 651-660.

Glantschnig, H., Rodan, G.A., and Reszka, A.A. 2002. Mapping of MST1 kinase sites of phosphorylation: Activation and autophosphorylation. I. Biol. Chem. 277: 42987-42996.

Graves, J.D., Gotoh, Y., Draves, K.E., Ambrose, D., Han, D.K., Wright, M., Chernoff, J., Clark, E.A., and Krebs, E.G. 1998. Caspase-mediated activation and induction of apoptosis by the mammalian Ste20-like kinase Mst1. ЕMBO J. 17: 2224-2234.

Hanahan, D. and Weinberg, R.A. 2000. The hallmarks of cancer. Cell 100: 57-70.

Hirai, S., Katoh, M., Terada, M., Kyriakis, J.M., Zon, L.I., Rana, A., Avruch, J., and Ohno, S. 1997. MST/MLK2, a member of the mixed lineage kinase family, directly phosphorylates and activates SEK1, an activator of c-Jun $\mathrm{N}$-terminal kinase/stress-activated protein kinase. J. Biol. Chem. 272: 15167-15173.

Jiang, J. and Struhl, G. 1995. Protein kinase A and Hedgehog signalling in Drosophila limb development. Cell 80: 563-572.

—. 1998. Regulation of the Hedgehog and Wingless signalling pathways by the F-box/WD40-repeat protein Slimb. Nature 391: 493-496.

Justice, R.W., Zilian, O., Woods, D.F., Noll, M., and Bryant, P.J. 1995. The Drosophila tumor suppressor gene warts encodes a homolog of human myotonic dystrophy kinase and is required for the control of cell shape and proliferation. Genes \& Dev. 9: 534-546.

Lee, K.K. and Yonehara, S. 2002. Phosphorylation and dimerization regulate nucleocytoplasmic shuttling of mammalian STE20-like kinase (MST). J. Biol. Chem. 277: 12351-12358.

Lee, K.K., Murakawa, M., Nishida, E., Tsubuki, S., Kawashima, S., Sakamaki, K., and Yonehara, S. 1998. Proteolytic activation of MST/Krs, STE20-related protein kinase, by caspase during apoptosis. Oncogene 16: 3029-3037.

Lee, K.K., Ohyama, T., Yajima, N., Tsubuki, S., and Yonehara, S. 2001. MST, a physiological caspase substrate, highly sensitizes apoptosis both upstream and downstream of caspase activation. J. Biol. Chem. 276: $19276-19285$

Newsome, T.P., Asling, B., and Dickson, B.J. 2000. Analysis of Drosophila photoreceptor axon guidance in eye-specific mosaics. Development 127: 851-860.

Richardson, H., O'Keefe, L.V., Marty, T., and Saint, R. 1995. Ectopic cyclin $\mathrm{E}$ expression induces premature entry into $\mathrm{S}$ phase and disrupts pattern formation in the Drosophila eye imaginal disc. Development 121: 3371-3379.

Robbins, D.J., Nybakken, K.E., Kobayashi, R., Sisson, J.C., Bishop, J.M., and Therond, P.P. 1997. Hedgehog elicits signal transduction by means of a large complex containing the kinesin-related protein costal2. Cell 90: 225-234.

Ryoo, H.D., Bergmann, A., Gonen, H., Ciechanover, A., and Steller, H. 2002. Regulation of Drosophila IAP1 degradation and apoptosis by reaper and ubcD1. Nat. Cell. Biol. 4: 432-438.

St. John, M.A., Tao, W., Fei, X., Fukumoto, R., Carcangiu, M.L., Brownstein, D.G., Parlow, A.F., McGrath, J., and Xu, T. 1999. Mice deficient of Lats1 develop soft-tissue sarcomas, ovarian tumours and pituitary dysfunction. Nat. Genet. 21: 182-186.

Stowers, R.S. and Schwarz, T.L. 1999. A genetic method for generating Drosophila eyes composed exclusively of mitotic clones of a single genotype. Genetics 152: 1631-1639.
Tapon, N., Harvey, K.F., Bell, D.W., Wahrer, D.C., Schiripo, T.A., Haber, D.A., and Hariharan, I.K. 2002. salvador promotes both cell cycle exit and apoptosis in Drosophila and is mutated in human cancer cell lines. Cell 110: 467-478.

Wolff, T. 2000. Histological techniques for the Drosophila eye. In Drosophila protocols (eds. W. Sullivan, et al.), pp. 201-272. Cold Spring Harbor Laboratory Press, Cold Spring Harbor, NY.

Wolff, T. and Ready, D.F. 1993. Pattern formation in the Drosophila retina. In The development of Drosophila melanogaster (eds. B. Bate and A. Martines Arias), pp. 1277-1325. Cold Spring Harbor Laboratory Press, Cold Spring Harbor, NY.

Xu, T., Wang, W., Zhang, S., Stewart, R.A., and Yu, W. 1995. Identifying tumor suppressors in genetic mosaics: The Drosophila lats gene encodes a putative protein kinase. Development 121: 1053-1063.

Yoo, S.J., Huh, J.R., Muro, I., Yu, H., Wang, L., Wang, S.L., Feldman, R.M., Clem, R.J., Muller, H.A., and Hay, B.A. 2002. Hid, Rpr and Grim negatively regulate DIAP1 levels through distinct mechanisms. Nat. Cell. Biol. 4: 416-424. 


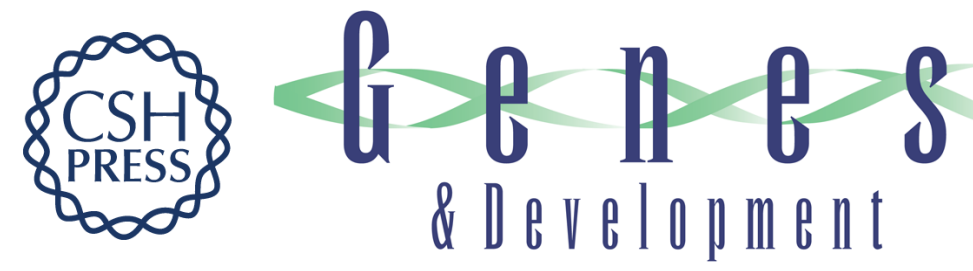

\section{The Drosophila Ste20 family kinase dMST functions as a tumor suppressor by restricting cell proliferation and promoting apoptosis}

Jianhang Jia, Wensheng Zhang, Bing Wang, et al.

Genes Dev. 2003, 17:

Access the most recent version at doi:10.1101/gad.1134003

References

This article cites 26 articles, 12 of which can be accessed free at:

http://genesdev.cshlp.org/content/17/20/2514.full.html\#ref-list-1

License

Email Alerting

Receive free email alerts when new articles cite this article - sign up in the box at the top

Service right corner of the article or click here.

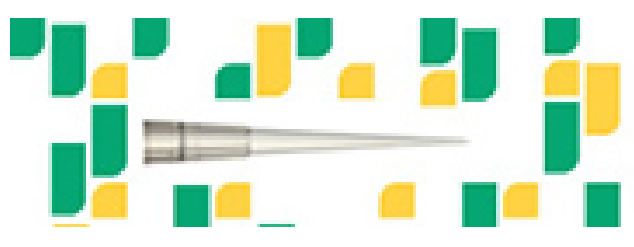

Focused on your science. 